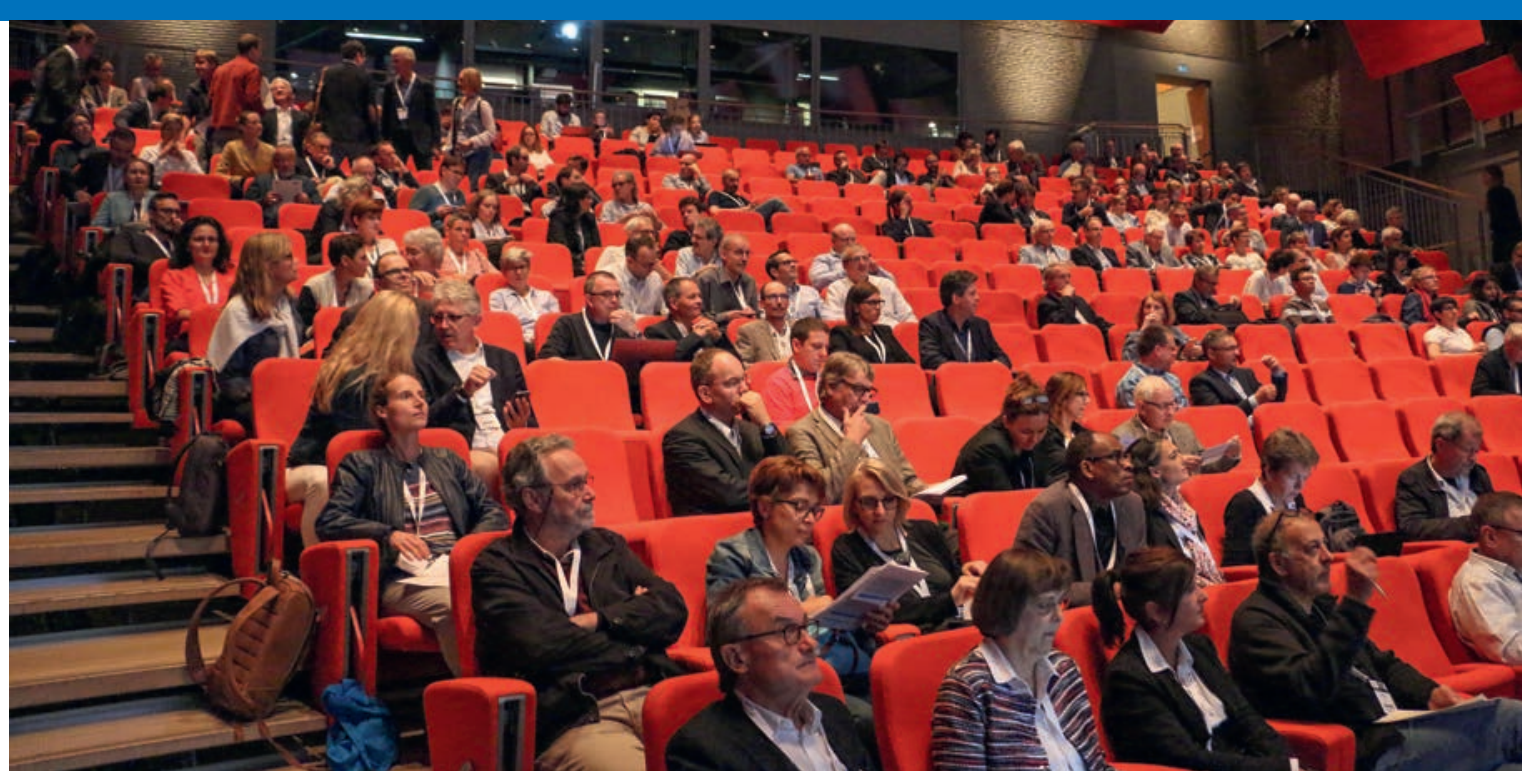

Das MedEd-Symposium 2019 im Zentrum Paul Klee in Bern bot dem Publikum ein vielfältiges Programm.

\title{
Von Megatrends und Wendepunkten
}

\section{Bruno Kesseli}

Dr. med. et lic. phil., Arzt und Medizinjournalist

Auch in seiner sechsten Auflage stiess das mittlerweile gut etablierte MedEd-Symposium des Schweizerischen Instituts für ärztliche Weiter- und Fortbildung (SIWF) zu den Perspektiven der ärztlichen Bildung auf grosses Interesse. Es fand zum dritten Mal in Folge im Zentrum Paul Klee statt und brachte interessante, teilweise auch überraschende Informationen, Kommentare und Denkanstösse zu den Perspektiven der ärztlichen Bildung.

Der neue Teilnehmerrekord von deutlich über 200 Symposiumsbesuchern in diesem Jahr kann als kleines Jubiläumsgeschenk für Werner Bauer gesehen werden. Das SIWF feiert 2019 sein 10-jähriges Bestehen,

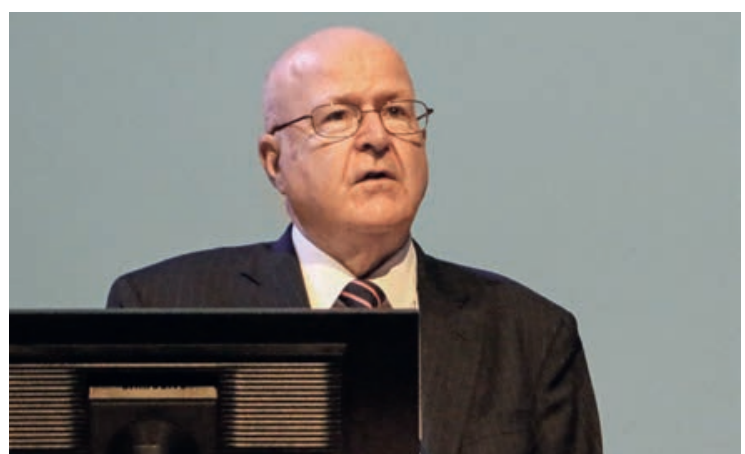

Werner Bauer. und Werner Bauer steht als Präsident auch bereits in seinem 10. Amtsjahr. Er unterstrich in seiner Begrüssungsansprache, dass das SIWF sich keineswegs auf Verwaltungsaufgaben beschränken, sondern bei der Gestaltung der ärztlichen Weiter- und Fortbildung eine aktive Rolle einnehmen wolle. Der Ärzteschaft hafte nicht ganz zu Unrecht der Ruf an, vorwiegend reaktiv zu sein. In diesem Punkte gelte es, Gegensteuer zu geben, wenn man für die Ärztinnen und Ärzte mehr erreichen wolle.

\section{Grusswort des BAG-Direktors}

$\mathrm{Zu}$ einer festen Grösse am MedEd-Symposium ist in den vergangenen sechs Jahren Pascal Strupler geworden. Der Direktor des Bundesamts für Gesundheit 


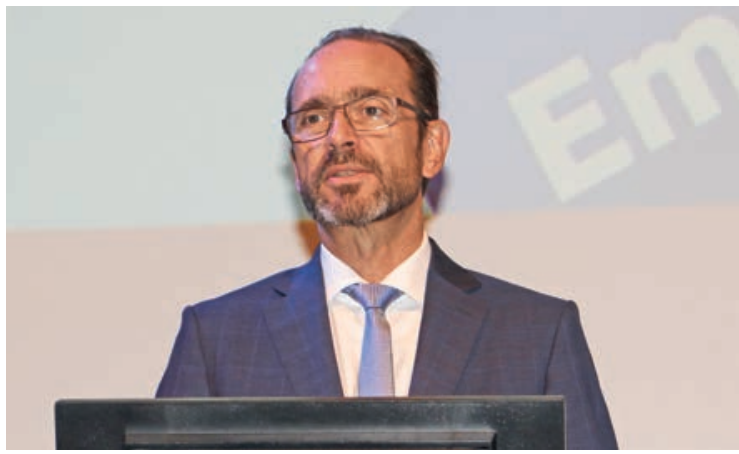

Pascal Strupler.

richtete wie stets seit der ersten Durchführung ein Grusswort ans Publikum. Ganz in der Tradition seiner früheren Ansprachen war er dabei sehr freundlich zur Ärzteschaft, lobte die Qualität der medizinischen Versorgung in der Schweiz, setzte aber auch ein paar feine Nadelstiche. Angesichts der aktuellen Entwicklungen müsse man sich mit der Frage beschäftigen, ob das heutige Weiterbildungssystem noch adäquat sei. Die neue Generation der Ärztinnen und Ärzte ticke anders als die vorangegangenen. Teilzeitarbeit stehe im Vordergrund, die Leitungsambitionen seien gering. Die beruflichen Anforderungen seien aber nach wie vor hoch. Somit sei es ein ebenso anspruchsvolles wie vordringliches Ziel, die veränderten individuellen Bedürfnisse mit den Ansprüchen der Gesellschaft an das Gesundheitswesen in Einklang zu bringen.

\section{Arztberuf und Megatrends}

Faktenbasiert-optimistisch ging es im Referat [1] von Daniel Dettling weiter. Der Jurist und Verwaltungswissenschaftler demonstrierte zunächst anhand einiger Beispiele aus dem Global Ignorance Test, dass wir einen Hang zum Awfulizing haben, also die Welt tendenziell schlechter machen, als sie ist. Auch das MedEdPulikum tappte bei der Schätzung der globalen Lebenserwartung in die Falle. Die Hirnforschung, so Dettling,

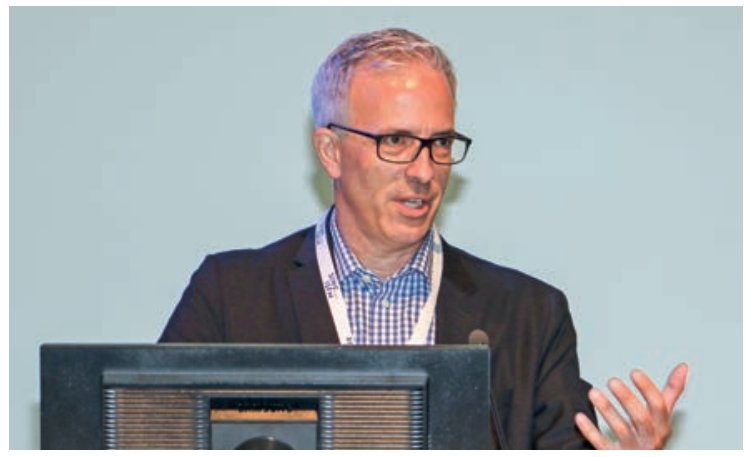

Daniel Dettling. liefere plausible Erklärungen für die Kluft zwischen Wahrnehmung und Wirklichkeit. Die Angst vor Gefahren und Zurückweisung habe evolutionsgeschichtlich klare Überlebensvorteile gebracht.

Wie die Medizin der Zukunft aussehen werde, sei unklar. Aber dass Menschlichkeit und Empathie im Umgang mit Patientinnen und Patienten zentral sein werden, steht für Dettling ausser Frage. Humanity werde immer wichtiger, und sie sei nicht automatisierbar. Deswegen führe die Digitalisierung letztlich zur Aufwertung des Arztberufes. Ärztinnen und Ärzte würden zunehmend zu Coaches ihrer Patienten. Künstliche Intelligenz sei dabei keine Konkurrenz, sondern diene der Erhöhung der menschlichen Intelligenz. Klagen drohten in Zukunft vor allem Ärzten, die schlecht kommunizierten. Elektronische Hilfsmittel werden gemäss Dettling weiter an Bedeutung zunehmen. Rund ein Drittel der Konsultationen finde in absehbarer Zeit nicht mehr in der Praxis statt. Das Smartphone werde zum virtuellen Leibarzt der Patienten. Dennoch scheint die Angst davor, dass Menschen im Gesundheitswesen durch Maschinen ersetzt werden, unbegründet. Bis zu 65\% neuer Jobs werden bis 2027 prognostiziert, darunter bisher kaum etablierte wie Gesundheitscoach, Bildungsberater, Wissens-Navigator oder persönlicher Health-Assistent. Gesundheitskompetenz, schloss Dettling seinen Tour d'Horizon, werde zu einer Schlüsselkompetenz des 21. Jahrhunderts.

\section{Sex und Gender in der Medizin}

Dass die genderspezifische Medizin innerhalb der gesamten Medizin ein Mauerblümchendasein fristet, führte Catherine Gebhard dem Publikum unter dem Titel «Sex und Gender in der Medizin: Konsequenzen für die Weiterbildung» eindrücklich vor Augen. Die Professorin für Kardiovaskuläre Gendermedizin und kardiale Bildgebung der Universität Zürich und des UniversitätsSpitals befasst sich seit Jahren intensiv mit dem Thema. Wie sie ausführte, ist es deshalb so wichtig, weil die wissenschaftliche Forschung klare Belege dafür liefert, dass eine One-fits-all-Medizin in der Regel nicht oder nur unbefriedigend funktioniert und zudem zu viele unerwünschte Wirkungen mit sich bringt. Gerade in der Kardiologie, aber nicht nur dort, lassen sich geschlechterspezifische Unterschiede an verschiedensten Beispielen mit harten Daten zeigen. Generell sind die Konsequenzen einer genderunspezifischen Medizin gravierend. Viele Wirkstoffe wurden oder werden nur an Männern getestet, sogar Hormonpräparate, die fast ausschliesslich von Frauen verwendet werden. Gemäss Zahlen der US-Arzneimittelbehörde traten bei Frauen in den USA zwischen 2004 und 


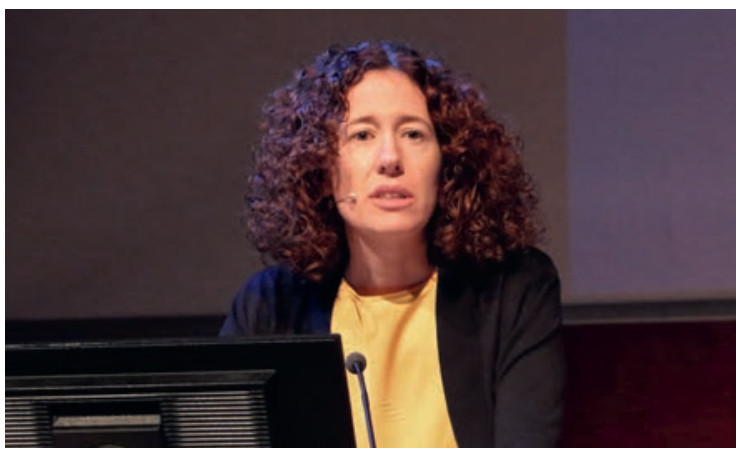

Catherine Gebhard.

2013 nach Medikamenteneinnahme über 50\% mehr unerwünschte Wirkungen auf als bei Männern. Acht von zehn Medikamenten werden wegen Nebenwirkungen vom Markt genommen, die ausschliesslich oder überwiegend bei Frauen auftreten. Obwohl Frauen entgegen der landläufigen Meinung häufiger an den Folgen einer kardiovaskulären Erkrankung sterben als Männer, erhalten sie beispielsweise bei Schlaganfällen weniger rasch medizinische Hilfe. Dies hängt auch damit zusammen, dass sich die Symptomatik kardiovaskulärer Ereignisse bei Frauen oft anders präsentiert als bei Männern. Umgekehrt werden Essstörungen und Depressionen bei Männern häufig nicht diagnostiziert, weil man diese Krankheitsbilder eher mit Frauen in Verbindung bringt.

Vereinzelte Silberstreifen zeichnen sich insofern am Horizont ab, als das Bewusstsein für die Vernachlässigung von geschlechts- und genderspezifischen Aspekten in der Medizin in den letzten Jahren zugenommen hat, sowohl innerhalb des Fachs selbst als auch - medial vermittelt - in der Öffentlichkeit. Fix the knowledge lautet für Catherine Gebhard das Motto, um die

\section{SIWF Award 2019: Die Ausgezeichneten}

Im Rahmen des 6. MedEd-Symposiums wurden auch die Trägerinnen und Träger des SIWF Award 2019 für besonderes Engagement in der Weiterbildung bekanntgegeben. Diese Auszeichnung wird aufgrund der Nomination durch ehemalige Assistenzärztinnen und -ärzte vergeben. Folgende Weiterbildende und Teams erhielten in diesem Jahr eine Auszeichnung: Einzelpersonen: Prof. Dr. med. Hatem Alkadhi; Dr. med. Alessandra Angelini; PD Dr. med. Johannes Dominik Bastian; Dr. med. Stefan Brodmann; Dr. med. Anna Giulia Brunello; Dr méd. Francesco Caronni; Dr. med. Stefan Eisoldt; PD Dr. med. Manuel Fischler; Dr. med. Sonia Frick; Dr méd. Paolo Gaffurini; Monsieur Gaël Grandmaison; Dr. med. Priska Grünig; Dr. med. Tamara Guidi; Dr. med. Thomas Herren; Dr. med. Ann-Kristin Hörsting; Prof. Dr. Stefan Klöppel; Herr Jens-Peter Kögel; Dr. med. David Lorenzana; Dr. med. Luzia Meier; Dr méd. François Pilet; Dr. med. Daniel Ribeiro; Dr. med. Florian Riese; Herr Bruno Rodrigues; Dr. med. Susanne Schibli; Prof. Dr. med. Torsten Schlote; Dr. med. Robert Schorn; Dr. med. Michael Studhalter; Prof. Dr. med. MichaelThiel; Dr. med. Annina Vischer.

Teams:Team Universitätsspital Basel, Radiologie; Team Frauenpraxis runa GmbH; Team Hirslanden Klinik St. Anna, Klinik für Innere Medizin und Nephrologie;Team SpitalThusis, Innere Medizin; Team Zentrum für Innere Medizin Aarau; Team Stadtspital Waid, Institute für Radiologie und Nuklearmedizin.

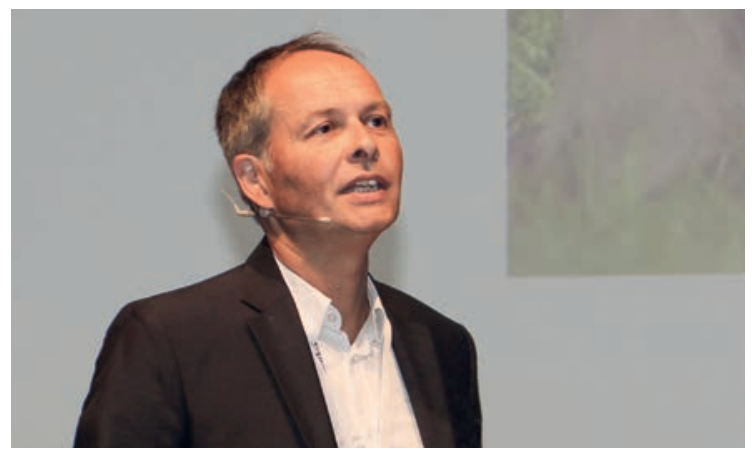

Daniel Genné.

Defizite zu verbessern. An diesem Leitspruch müssen sich sowohl die Forschung als auch die Medical Education und die Patientenversorgung orientieren.

\section{Licht- und Schatten der Weiterbildung}

Auf die drei parallel geführten Seminare [2], mit denen der MedEd-Nachmittag traditionellerweise eröffnet wird, folgte die letzte Keynote des Tages. Daniel Genné, Chefarzt und Spitalleitungsmitglied des Spitalzentrums Biel, vermittelte dem Publikum seine Sicht auf «Die Weiterbildung heute: Licht und Schatten». Er machte deutlich, dass die Qualität der ärztlichen Weiterbildung von einer Vielzahl von "volatilen" Faktoren beeinflusst wird. Eine Schlüsselfunktion haben die Weiterbildenden, die ihrerseits eine Vielzahl von Rollen zu erfüllen haben, darunter neben den traditionellen - Arzt, Forschender und Lehrer - auch arztfernere wie Manager, Finanzexperte oder Kommunikationsbeauftragter.

Weitere «Stressfaktoren» für die Weiterbildung sind der ökonomische Druck, die Zunahme administrativer Aufgaben und die Beschränkung der Arbeitszeit auf 50 Wochenstunden. Basierend auf Umfragen bestätigte Genné die Aussage von Pascal Strupler, dass die Generation Y «anders tickt». Die Work-Life-Balance ist für sie essentiell, Teilzeitarbeitsmöglichkeiten, Auszeiten für private Projekte, Sabbaticals oder auch 24-Stunden-Kitas sind ihnen wichtig. Dazu kommen hohe Ansprüche an ihre Vorgesetzten, von denen sie ein ausgezeichnetes Fachwissen, regelmässige und zeitnahe Feedbacks, intensives Coaching und eine Reduktion des administrativen Aufwands erwarten.

Diese komplexe Ausgangslage macht die Organisation einer guten Weiterbildung zu einer sehr anspruchsvollen und schwierig zu finanzierenden Aufgabe. Ohne das Ei des Kolumbus aus dem Hut zaubern zu können, präsentierte Genné eine Reihe interessanter Überlegungen und Vorschläge zur Sicherung oder Verbesserung der Qualität in der Weiterbildung. Die EPAs (Entrustable Professional Activities) hält er für ein gutes 


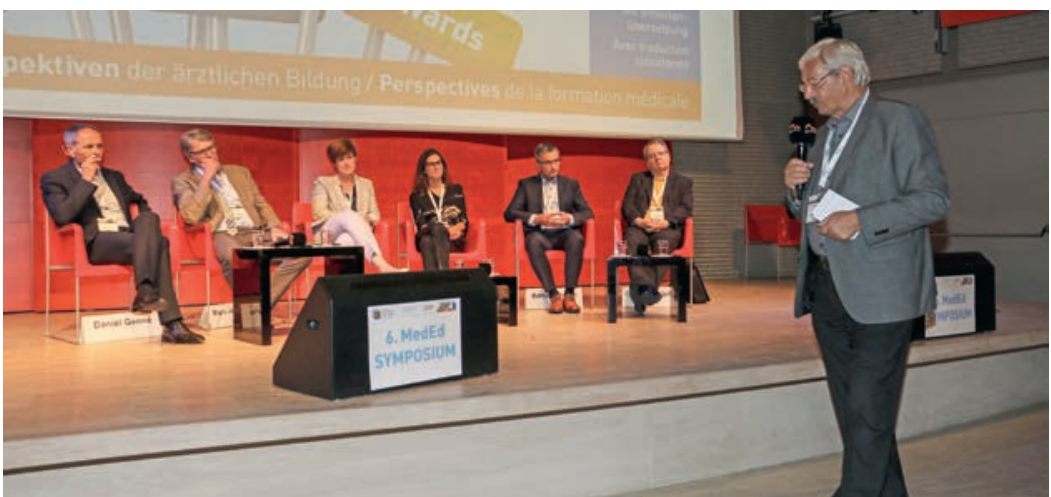

Unter der Leitung von Iwan Rickenbacher (ganz rechts) diskutierten (von links): Daniel Genné, Markus Furrer, Bernadette Häfliger Berger, Dina-Maria Jakob, Adrian Göldlin, Guido Schüpfer.

Instrument, sofern sie in intelligenter Weise in die Weiterbildungsprogramme integriert werden. Eine Verbesserung der Weiterbildungsqualität verspricht er sich durch die Einführung einer Art von Tansfermarkt für Kaderärztinnen und -ärzte, ähnlich wie dies im Profifussball gang und gäbe ist. Dabei würde die Weiterbildungsqualität der betreffenden Ärztinnen und Ärzte nach einem objektivierbaren Punkteschema bewertet und die sie verpflichtenden Ausbildungsspitäler erhielten entsprechende finanzielle Unterstützung, wobei das Geld ausschliesslich in die Weiterbildung fliessen dürfte. Genné zeigte sich allerdings überzeugt, dass die besten Konzepte wenig bringen, wenn an einer Weiterbildungsstätte kein gutes Arbeitsklima herrscht. Ein solches zu schaffen, war und ist ihm auch an seiner eigenen Wirkstätte ein zentrales Anliegen.

\section{Bildnachweis \\ Bruno Kesseli}

b.kesseli@hispeed.ch

\section{Lebhaftes Podium}

Befindet sich die «Weiterbildung an einem Wendepunkt?». Zu dieser Frage diskutierten unter der Leitung von Iwan Rickenbacher zum Abschluss der Veranstal-

\section{SIWF-Projektförderung 2019}

Die Einladung, Projekte zur Unterstützung der Weiterbildung einzureichen, hat erneut ein überraschend grosses Echo ausgelöst. Aus den 47 eingegangenen Weiterbildungsprojekten hat die Jury fünf Projekte ausgezeichnet, die durch das SIWF mit einem Förderungsbeitrag unterstützt werden (insgesamt 150000 Franken). Die Projekte wurden am MedEd-Symposium kurz vorgestellt:

- prEPAred - Mehr Präzision in der Weiterbildung durch Entwicklung eines EPA-basierten Assessmentsystems (UniversitätsSpital Zürich);

- Einsatz von Multisource-Feedback in der ärztlichen Weiterbildung: Erstellung einer Leitlinie zur Implementierung (Universitäts-Kinderspital Zürich);

- Spital-Wiki (Spital Linth);

- EPA: Just do it! A faculty development project to implement postgraduate EPAS in the clinical setting in Switzerland (Spital Lachen);

- Einführung des videobasierten 4-Stufen-Modells von Peyton zur Anleitung von Assistenzärzten am Beispiel der Nabelvenenkathetereinlage - eine randomisiert kontrollierte Studie (Universitäts-Kinderspital beider Basel) tung fünf Podiumsgäste aus verschiedenen Sparten der Medizin und die Leiterin der Abteilung Gesundheitsberufe des BAG [3]. Als potenzielle Wendepunkte wurden die Interprofessionalität und die Finanzierungsprobleme der Weiterbildung genannt, aber auch neue Rollen, in die sich Ärztinnen und Ärzte begeben müssten. Multi-Tasking-Persönlichkeiten seien in $\mathrm{Zu}$ kunft gefragt, zeigte sich Daniel Genné überzeugt. Markus Furrer bemängelte, dass in der Medizin die Durchlässigkeit fehle. Einer Pflegefachfrau sei es auch bei entsprechender Befähigung nicht möglich, sich bei Ärztemangel in einem erleichterten Verfahren zur Ärztin weiterzubilden. Bernadette Häfliger wies darauf hin, dass das Medizinalberufegesetz solche Abkürzungen zurzeit nicht erlaube. Auf der Plattform ärztliche Bildung werde aber intensiv über neue Modelle und Kompetenzen nachgedacht.

Viele Voten drehten sich um die Finanzierung der Weiterbildung. Zehn Jahre Unterfinanzierung der Weiterbildung zeigten Wirkung, meinte Guido Schüpfer. Am Ende gehe es eben doch immer ums Geld. Die Runde war sich aber einig, dass die Weiterbildung zum Selbstverständnis der Spitäler gehören müsse.

Werner Bauer zeigte sich in seinem Schlusswort verhalten optimistisch, was die zukünftige Finanzierung der Weiterbildung angeht - immerhin habe er von Spital-CEOs und Polit-Funktionären positive Signale erhalten. Sein Schlussfazit war allerdings glasklar: «Osler hätte heute als Chefarzt keine Chance» [4].

\section{Literatur}

1 Die Präsentationen zu den Hauptreferaten und Seminaren sind zugänglich unter: www.siwf.ch/siwf-projekte/meded-symposium.cfm

2 Seminar A: Didaktik in der Medizin: Tipps und Tricks für Weiterbildende. Dr. med. Christian Schirlo, Leitung Geschäftsbereich Struktur \& Entwicklung, Geschäftsstelle Direktorium UMZH, Zürich; Dipl. Psych. Anja Pawelleck, Teamleiterin Hochschuldidaktische Weiterbildung UZH, Zürich; Dr. med. Urs Strebel, ehem. Chefarzt Medizin, Spital Männedorf; Dr. med. Andrea Meienberg, Trainee «RCP Workshop Facilitator», Universitätsspital Basel. Seminar B: Entrustable Professional Activities (EPA's). Dr. med. Regula Schmid, Leitende Ärztin Neuropädiatrie, Kantonsspital Winterthur; Dr. med. Sonia Frick, MME, Leitende Ärztin Innere Medizin, Spital Lachen; Dr. med. Adrian Marty, MME, Medizinischer Leiter Simulationszentrum UniversitätsSpital Zürich.

Seminar C: Burnout des étudiants(es) et médecins assistants(es). Dr méd. Nadia Bajwa, MD, MHPE Université de Genève, Faculté de Médecine, Hôpitaux Universitaires de Genève; Prof. Dr méd. Raphaël Bonvin, Université de Fribourg, Pédagogie Médicale; Dr méd. Anja Zyska Cherix, MD, Présidente de l'ASMAC-VSAO.

3 Die Experten auf dem Podium: Prof. Dr méd. Daniel Genné, Médecin-chef de la Clinique de Médecine et Membre de la Direction, Centre hospitalier Bienne; Dr. med. Adrian Göldlin, Sanacare Bern; Bernadette Häfliger Berger, RA, MAS NPPM, Leiterin Abteilung Gesundheitsberufe, Bundesamt für Gesundheit (BAG), Bern; Prof. Dr. med. Jürg Hodler, Ärztlicher Direktor, UniversitätsSpital, Zürich; Dr. med. Dina-Maria Jakob; Resort Weiterbildung Verband Schweizer Assistenz- und Oberärztinnen und -ärtze (VSAO); Dr. med. Guido Schüpfer, Leiter Stab Medizin, Kantonsspital Luzern.

4 Der von Werner Bauer hochgeschätzte und gern zitierte Sir William Osler, 1849-1919, war ein kanadischer Mediziner, Physiologe und Medizinhistoriker. Wegen seiner bahnbrechenden Lehrmethoden wird er häufig als Vater der modernen Medizin bezeichnet. 